\title{
AN ATTEMPT TO DETECT THE 327-MC/S LINE OF GALACTIC DEUTERIUM
}

\author{
R. L. ADGIE \\ Royal Radar Establishment, Malvern, England
}

1. INTRODUCTION

The previous unsuccessful attempts to detect the $327-\mathrm{Mc} / \mathrm{s}$ line of galactic deuterium $[1,2]$ were limited in sensitivity either by the size of aerial or by the inability to use long integration times with a large fixed aerial. The new observations reported here were made with the 250-foot radio telescope at Jodrell Bank, which can continuously follow a source of radiation ; moreover, the effective integration time was improved by using a multi-channel receiver instead of a swept-frequency one.

The received signal intensity can be increased by searching for the line in absorption instead of in emission, with the aerial directed toward an intense source of continuous radiation. If the intervening gas has an optical depth $\tau$ and the intense source produces an aerial temperature $T_{A}$, then the line intensity is $\tau T_{A}$, whereas the same gas in emission would produce a line intensity in the aerial of less than $\tau T_{K}$, where $T_{K}$ is the kinetic temperature of the gas. At this low frequency, $T_{A}$ can be much larger than $T_{K}$ (about $\left.100^{\circ} \mathrm{K}\right)$, especially with a large aerial, giving a considerable increase in signal. But $T_{A}$ also increases the total noise, and it is the signal-to-noise power ratio $(\mathrm{S} / \mathrm{N})$ that determines the limit of sensitivity. Therefore, in absorption,

$$
\mathrm{S} / \mathrm{N}=\frac{\tau T_{A}}{T_{A}+T_{R}}
$$

where $T_{R}$ is the receiver noise temperature. When $T_{A}$ is much larger than $T_{R}$ the $\mathrm{S} / \mathrm{N}$ ratio reaches the limiting value $\tau$, which cannot be increased by using a larger aerial. This limit was reached when observing the intense source Cassiopeia $\mathrm{A}$ with the 250-foot radio telescope.

It is also worth considering the $\mathrm{S} / \mathrm{N}$ ratio that could be obtained in emission using a very low noise receiver (e.g., maser). The total noise is now a combination of aerial temperature produced by the galactic background and the total effective receiver noise; therefore, for emission,

$$
\mathrm{S} / \mathrm{N}=\frac{\tau T_{\mathrm{K}}}{T_{\theta}+T_{R}}
$$

In the galactic plane $T_{\theta}$ is about $50^{\circ} \mathrm{K}$ away from the galactic center, and if feeder losses and ground radiation are taken into account $T_{R}$ will also be about $50^{\circ} \mathrm{K}$. This gives a limiting $\mathrm{S} / \mathrm{N}$ ratio of $\tau$ if observations are made 
with a large aerial having a beamwidth less than the angular size of the gas. The advantage of this method is that observations are not limited to the positions of the intense sources and it may be possible to find other positions that have larger optical depths giving larger $\mathrm{S} / \mathrm{N}$ ratios.

\section{EXPERIMENTAL RESULTS}

The receiver has 11 channels, separated by $18 \mathrm{kc} / \mathrm{s}$, each one having an $18 \mathrm{kc} / \mathrm{s}$ bandwidth, which corresponds to the line-width derived from the hydrogen-line absorption measurements on Sagittarius A [3]. Observations were also made on Cassiopeia $\mathrm{A}$, tuning the receiver to the frequency of the Perseus arm corresponding to $-45 \mathrm{~km} / \mathrm{second}[4,5]$. Only the center channel coincides with the expected deuterium line; the others determine the zero level and check for interference. The total integrating time on each of these sources was 18 hours, which corresponds to an increase in the $\mathrm{S} / \mathrm{N}$ ratio through the receiver of $5 \times 10^{4}$.

If the sensitivity limit is taken as twice the R.M.S. value of the residual fluctuations, then the derived upper limits for the optical depth of deuterium are $0.7 \times 10^{-4}$ and $1.0 \times 10^{-4}$ for Cassiopeia $\mathrm{A}$ and Sagittarius A, respectively. The hydrogen-line optical depth averaged over the bandwidth used is about 1.3 for both these sources. From the equation

$$
\frac{\tau_{\mathrm{H}}}{\tau_{\mathrm{D}}}=\frac{9}{8} \frac{\nu_{\mathrm{H}}}{\nu_{\mathrm{D}}} \frac{n_{\mathrm{H}}}{n_{\mathrm{D}}}
$$

the abundance ratio can be evaluated, giving limits for the ratio of hydrogen to deuterium as $4 \times 10^{3}$ and $2.5 \times 10^{3}$ for Cassiopeia A and Sagittarius A.

This result obtained for Sagittarius A appears to represent only a slight improvement on the value previously reported [1], but this is because of an overestimation in the original experiment of the expected optical depth of the hydrogen between the sun and the galactic center. At that time the discrete source Sagittarius A was thought to be less than 3 kiloparsecs from the sun [4], and the hydrogen-line optical depth found from absorption measurements on this source was increased by 3 times to give the expected value from the sun to the galactic center, since the galactic radiation concentrated toward the center formed the major portion of the continuous radiation received at the deuterium-line frequency. The present results have been evaluated on the assumption that Sagittarius $\mathrm{A}$ is at the galactic center [6], and no correction has been applied to the observed hydrogen-line optical depth.

Although the experiment has failed to detect the presence of deuterium in the Galaxy it seems reasonable to conclude from the results that the ratio of deuterium to hydrogen is probably not greater than the terrestrial value of $1.5 \times 10^{-4}$ [7].

This work forms part of the radio astronomy program of Dr. J. S. Hey's group at the Royal Radar Establishment; we are much indebted to Professor A.C. B. Lovell for making available the 250 -foot radio telescope for this experiment. 


\section{REFERENCES}

[1] Adgie, R. L., and Hey, J. S. Nature, 179, 370, 1957.

[2] Stanley, G. J., and Price, R. Nature, 177, 1221, 1956.

[3] McClain, E. F. Ap. J. 122, 376, 1955.

[4] Hagen, J. P., Lilley, A. E., and McClain, E. F. Ap. J. 122, 361, 1955.

[5] Muller, C. A. Ap. J. 125, 830, 1957.

[6] van Woerden, H., Rougoor, G. W., and Oort, J. H. C.R. 244, 1691, 1957.

[7] Suess, H. E., and Urey, H. C. Rev. Mod. Phys. 28, 53, 1956.

\section{Discussion}

Stanley: I would like to point out that the value of $T_{R}\left(50^{\circ} \mathrm{K}\right)$ used by Adgie represents the current state of techniques. There is every reason to believe that much lower values of $T_{R}$ might be obtained.

Adgie: My estimate of $50^{\circ} \mathrm{K}$ for $T_{R}$ in the case of a maser includes feeder losses and ground radiation. And even if $T_{R}$ could be reduced to a few degrees there is still about $50^{\circ} \mathrm{K}$ at the aerial from the galactic radiation. It might therefore be possible to obtain an $\mathrm{S} / \mathrm{N}$ of $2 \tau$ under ideal conditions, which is only a factor-of- 2 improvement over the absorption measurements.

Hoyle: It is important to stress that that this experiment is of great importance to studies of nucleogenesis. Even negative results are important. It would be significant if one could conclude, for example, that the deuterium abundance is definitely less in the interstellar gas than it is on the earth.

Conway: Perhaps it is worth recalling that on the earth the abundance ratio of deuterium to hydrogen is 1 to 5000 , just less than the upper limit found by Adgie. 